We suggest that a previously conjectured relation between Structure Functions (SF) for nuclei and nucleons also links distribution functions (df) for partons in a nucleus and in nucleons. The above suggestion ensures in principle identical results for $\mathrm{SF} F_{2}^{A}$, whether computed with hadronic or partonic degrees of freedom. In practice there are differences, due to different $F_{2}^{n}$ input. We show that the thus defined nuclear parton distribution functions (pdf) respect standard sumrules. In addition we numerically compare some moments of nuclear SF, and find agreement between results, using hadronic and partonic descriptions. We present computations of EMC ratios for both and compare those with hadronic predictions and data. In spite of substantial differences in the participating SF, the two representations produce approximately the same EMC ratios. The apparent correlation between the above deviations is ascribed to a sumrule for $F_{2}^{A}$. We conclude with a discussion of alternative approaches to nuclear pdf.

\title{
On Distribution Functions for Partons in Nuclei
}

\author{
A.S. Rinat and M.F Taragin \\ Weizmann Institute of Science, Department of Particle Physics, Rehovot 76100, Israel
}

(Dated: June 21, 2018)

\begin{abstract}
We suggest that a previously conjectured relation between Structure Functions (SF) for nuclei and nucleons also links distribution functions (df) for partons in a nucleus and in nucleons. The above suggestion ensures in principle identical results for $\mathrm{SF} F_{2}^{A}$, whether computed with hadronic or partonic degrees of freedom. In practice there are differences, due to different $F_{2}^{n}$ input. We show that the thus defined nuclear parton distribution functions (pdf) respect standard sumrules. In addition we numerically compare some moments of nuclear SF, and find agreement between results, using hadronic and partonic descriptions. We present computations of EMC ratios for both and compare those with hadronic predictions and data. In spite of substantial differences in the participating SF, the two representations produce approximately the same EMC ratios. The apparent correlation between the above deviations is ascribed to a sumrule for $F_{2}^{A}$. We conclude with a discussion of alternative approaches to nuclear pdf.
\end{abstract}




\section{INTRODUCTION.}

A large body of data is presently available on inclusive lepton scattering from nuclei and on subsequently extracted nuclear Structure Functions (SF). Standard treatments of those data use hadron degrees of freedom, whereas in an alternative approach, one employs df of partons in nuclei. In one of the first papers on EMC ratios, Akulinichev et al related df for quarks in a nucleon, nucleons in nuclei and quarks in nuclei by a generalized convolution, schematically written as [1] (see also Refs. [2, 3] )

$$
f_{q / A}=f_{N / A} * f_{q / N}
$$

Variations of the above and various approximations have since been the preferred tool for the analysis of EMC ratios, whether in terms of the hadron or a parton representation.

There exist alternative treatments of nuclear pdf. For instance Frankfurt and Strikman discuss nuclear pdf, but do not relate those to the pdf of a nucleon [4]. Other approaches parametrize information on $\mathrm{SF}$ at some scale $Q_{0}^{2}$, and then evolve to a desired $Q^{2}$. Data then determine the introduced parameters [5, 6]. Next we mention quark models for nuclei, which have been used in direct calculations of df. Their potential is presumably limited to the lightest nuclei 7]. Finally there are approaches, where the effect of a nuclear medium on a nucleon or a quark is replaced by mean fields [8, 9, 10].

In the present note we make a simple, nearly natural choice for nuclear pdf, which is free of adjustable parameters. Those satisfy basic sum rules and produce the same $F_{2}^{A}$ as computed from a hadronic base. In the conclusion we compare some of the above-mentioned alternative proposals with our choice.

\section{A FEW ESSENTAILS.}

We start with the cross section per nucleon for the scattering of unpolarized electrons with energy $E$ over an angle $\theta$

$$
\frac{d^{2} \sigma^{A}(E ; \theta, \nu)}{d \Omega d \nu}=\sigma_{M}(E ; \theta, \nu)\left[\frac{2 x M}{Q^{2}} F_{2}^{A}\left(x, Q^{2}\right)+\frac{2}{M} F_{1}^{A}\left(x, Q^{2}\right) \tan ^{2}(\theta / 2)\right]
$$

$\sigma_{M}$ is the Mott cross section and $F_{1,2}^{A}\left(x, Q^{2}\right)$ are the standard nuclear SFs per nucleon. Those depend on the squared 4-momentum transfer $q^{2}=-Q^{2}=-\left(|\boldsymbol{q}|^{2}-\nu^{2}\right)$ and on the Bjorken variable $0 \leq x=Q^{2} / 2 M \nu \leq A$ in terms of the nucleon mass $M$. 
Next we make explicit the specific relation (1.1) between nuclear and nucleonic SF [11]

$$
\begin{aligned}
F_{k}^{A}\left(x, Q^{2}\right) & =\int_{x}^{A} \frac{d z}{z^{2-k}} f^{P N, A}\left(z, Q^{2}\right) F_{k}^{\langle N\rangle}\left(\frac{x}{z}, Q^{2}\right) \\
F_{k}^{\langle N\rangle}=\left[Z F_{k}^{p}+N F_{k}^{n}\right] / 2 A & =\left[1-\frac{\delta N}{A}\right] F_{k}^{p}+\left[1+\frac{\delta N}{A}\right] F_{k}^{n}
\end{aligned}
$$

$F_{k}^{p, n}$ are SFs of $p, n$, whereas $F_{k}^{\langle N\rangle}$ defines a nucleon SF, obtained by weighting $F_{k}^{p, n}$ with $Z, N . \delta N / A$ denotes the relative neutron excess.

Our approach draws on the Gersch-Rodriguez-Smith (GRS) theory for inclusive scattering of non-relativistic projectiles [12], in which case the linking $f^{P N, A}$ is the SF of a fictitious nucleus, composed of point-like nucleons. Eq. (2.2) results from a covariant generalization of the GRS theory [13]. The theory is non-perturbative with on-mass shell nucleon SFs.

Eq. (1.1) has originally been formulated in the Bjorken limit 1]. We have postulated (2.2) to hold for finite $Q^{2}$ [11] and an extensive body of data in the ranges $x \gtrsim 0.2 ; Q^{2} \gtrsim 2.5 \mathrm{GeV}^{2}$ [14, 15] appears to be accounted for by the relation (2.2) [16, 17, 18].

Eq. (2.2) does not contain a term, which account for quarks in virtual mesons [19], and it also lacks (anti-)screening effects [20]. Those are negligible for $x \gtrsim 0.2$ and we limit ourselves to that range [44].

In the following it appears useful to separate the nucleon $\mathrm{SF} F_{k}^{N}=F_{k}^{N, N E}+F_{k}^{N, N I}$ into nucleon elastic and inelastic components, which correspond to absorption processes of a virtual photon on a $N, \gamma^{*}+N \rightarrow N$, (NE), or $\gamma^{*}+N \rightarrow$ (hadrons,partons) (NI). Elastic components for a $N$ are proportional to the standard combinations of squared electromagnetic form factors $G_{E, M}^{N}\left(Q^{2}\right)$ and vanish, unless $x=1$. With $\left[\tilde{G}^{\langle N\rangle}\right]^{2}=\left[Z\left(G^{p}\right)^{2}+\right.$ $\left.N\left(G^{n}\right)^{2}\right] / A$, one has

$$
\begin{aligned}
& F_{1}^{N, N E}\left(x, Q^{2}\right)=\frac{1}{2} \delta(1-x)\left[\tilde{G}_{M}^{\langle N\rangle}\left(Q^{2}\right)\right]^{2} \\
& F_{2}^{N, N E}\left(x, Q^{2}\right)=\delta(1-x) \frac{\left[\tilde{G}_{E}^{\langle N\rangle}\left(Q^{2}\right)\right]^{2}+\eta\left[\tilde{G}_{M}^{\langle N\rangle}\left(Q^{2}\right)\right]^{2}}{1+\eta}
\end{aligned}
$$

The corresponding nuclear NE (QE) components are from Eq. (2.2) seen to be

$$
\begin{aligned}
& \left.F_{1}^{A, N E}\left(x, Q^{2}\right)=\frac{f^{P N, A}\left(x, Q^{2}\right)}{2}\left[\tilde{G}_{M}^{\langle N\rangle}\left(Q^{2}\right)\right]^{2}\right] \\
& F_{2}^{A, N E}\left(x, Q^{2}\right)=x f^{P N, A}\left(x, Q^{2}\right) \frac{\left[\tilde{G}_{E}^{\langle N\rangle}\left(Q^{2}\right)\right]^{2}+\eta\left[\tilde{G}_{M}^{\langle N\rangle}\left(Q^{2}\right)\right]^{2}}{1+\eta}
\end{aligned}
$$


In particular for the lightest nuclei the normalized $f^{P N, A}$ peaks around $x \approx 1$, and the same holds for the above QE components $F_{k}^{A, N E}\left(x, Q^{2}\right)$. The above summarizes elements of a hadronic description of nuclear SF: we now turn to a partonic representation.

\section{A SIMPLE CHOICE FOR NUCLEAR PARTON DISTRIBUTION FUNC- TIONS.}

We start with the leading order twist contributions to the dominant NI components of nucleon SF for finite $Q^{2}$. With no danger of confusion we shall omit one or both arguments $x, Q^{2}$. Neglecting heavy quark contributions and decomposing quark $\mathrm{df} q=q_{v}+\bar{q}$ into valence and sea quarks parts, one has

$$
\begin{aligned}
& F_{2}^{p}=\frac{x}{9}\left(4 u_{v}+d_{v}+8 \bar{u}+2 \bar{d}+2 s\right) \\
& F_{2}^{n}=\frac{x}{9}\left(u_{v}+4 d_{v}+2 \bar{u}+8 \bar{d}+2 s\right)
\end{aligned}
$$

Similarly for a 'nucleon', defined as the $Z, N$ weighted $p, n$, one has

$$
F_{2}^{\langle N\rangle} \equiv \sum_{i} a_{i} x q_{i}=\frac{5 x}{18}\left[u_{v}+d_{v}+2 \bar{u}+2 \bar{d}+\frac{4}{5} s-\frac{3 \delta N}{5 A}\left(u_{v}-d_{v}+2 \bar{u}-2 \bar{d}\right)\right],
$$

Nuclear pdf ought to reproduce nuclear $\mathrm{SF} F_{k}^{A}$, just as proton pdf do for $F_{k}^{p}$, Eq. (3.1), but that necessary requirement is insufficient for their determination. In a construction we proceed in two steps. First we choose $F_{2}^{A}\left(q^{A}\right)$ to be the same combination of df of partons in a nucleus, as the above $F_{2}^{\langle N\rangle}$, Eq. (3.2), is for the averaged nucleon

$$
F_{2}^{A}=\sum_{i} a_{i} x q_{i}^{A}=\frac{5 x}{18}\left[u_{v}^{A}+d_{v}^{A}+2 \bar{u}^{A}+2 \bar{d}^{A}+\frac{4}{5} s^{A}-\frac{3 \delta N}{5 A}\left(u_{v}^{A}-d_{v}^{A}+2 \bar{u}^{A}-2 \bar{d}^{A}\right)\right]
$$

Upon substitution into Eq. (2.2), and using Eq. (3.2), one finds

$$
F_{2}^{A}=\sum_{i} a_{i}\left[f^{P N, A} x * q_{i}^{A}\right]
$$

Comparison with Eq. (3.3) still does not result in a unique expressions for each individual pdf $q_{i}^{A}$. Then guided by Eq. (2.2), we make the following second choice, with no flavor mixing of valence and sea quarks

$$
x q_{i}^{A}\left(x, Q^{2}\right) \equiv \int_{x}^{A} d z f^{P N, A}\left(z, Q^{2}\right)\left(\frac{x}{z}\right) u_{v}\left(\frac{x}{z}, Q^{2}\right)
$$




$$
\begin{aligned}
& x \bar{q}_{i}^{A}\left(x, Q^{2}\right) \equiv \int_{x}^{A} d z f^{P N, A}\left(z, Q^{2}\right)\left(\frac{x}{z}\right) d_{v}\left(\frac{x}{z}, Q^{2}\right) \\
& x g^{A}\left(x, Q^{2}\right) \equiv \int_{x}^{A} d z f^{P N, A}\left(z, Q^{2}\right)\left(\frac{x}{z}\right) g\left(\frac{x}{z}, Q^{2}\right)
\end{aligned}
$$

Eqs. (3.5) with one $f$ for all partons (in case for the PWIA) had already be suggested by Berger and Coester [2].

In view of the meager experimental information on non-valence parton distributions in nuclei, we shall also investigate changes when non-valence df are not affected by the nuclear medium (cf. [5, 6] ), thus

$$
\bar{q}^{A} \equiv \bar{q} ; s^{A}=\bar{s}^{A}=s ; g^{A}=g^{N}
$$

Eqs. (3.3), (3.5) (or alternatively Eq. (3.6)) manifestly produce the same $F_{2}^{A}$ in the parton and the hadronic representation, provided one uses exactly the same input $f^{P N, A}$ and $F_{2}^{\langle N\rangle}$ in both.

In practice this is not the case, in particular not for $F_{2}^{n}$ in $F_{2}^{\langle N\rangle}$. In the absence of direct information, the CteQ parametrizations exploit data on $F_{2}^{D}$. Using the 'primitive' approximation $F_{2}^{n}(x)=2 F_{2}^{D}(x)-F_{2}^{p}, \quad F_{2}^{n}(x)$ replaces $F_{2}^{D}$ as input. However, the above approximation deteriorates with $x$, increasing from $x \gtrsim(0.25-0.30)$. As we shall shortly demonstrate, its use for larger $x$ leads to misfits with data.

As to the options in the version CteQ6 [22] we selected the one, with $F_{2}^{p}$, closest to the Arneodo parametrization of resonance-averaged data [23]. This appears possible for $x \lesssim 0.6-0.7$. Using $S U_{3}$ symmetry one obtains in the usual way $F_{2}^{n}$ in terms of $q_{i}, \bar{q}_{i}$.

In contrast, in the hadronic approach one stays as close as possible to data. For not too high $Q^{2}$, one can exploit parametrizations of actual $F_{2}^{p}$ data [24]. However, the range $Q^{2} \gtrsim 3.5 \mathrm{GeV}^{2}$ of our interest borders the limits of validity of the data parametrizations, we chose to use the above-mentioned Arneodo representation of $F_{2}^{p}$ [23]. As to $F_{2}^{n}$, it has been obtained for all $x$ by an indirect extraction method (see for instance Ref. [25]). Fig. 1 for $C=F_{2}^{n} / F_{2}^{p}$ in the two representations, $Q^{2}=3.5,5.0 \mathrm{GeV}^{2}$, makes manifest the abovementioned differences, which increase with $x$ [45].

A second subtle difference between the representations is the validity of Eq. (2.2) and (3.5). The former one is explicitly limited to the nucleonic part of $F_{2}^{A}$, i.e. roughly for $x \gtrsim 0.2$, while the latter is conceivably valid out to lower $x$ (cf. Ref. 27). 
The above described freedom in the choice of df for partons in nuclei is not unlimited, since $\mathrm{df}$ are constrained by sum rules. We first check those, which are directly related to the normalization of $f^{P N, A}$ :

a) For any linear combination $C$ of $\mathrm{df}$ for valence quarks

$$
\begin{aligned}
\int_{0}^{A} d x C^{A} & =\int_{0}^{1} d x C^{N} \\
\int_{0}^{A} d x\left(u_{v}^{A}+d_{v}^{A}\right) & =\int_{0}^{1} d x\left(u_{v}+d_{v}\right)=3 \\
\int_{0}^{A} d x \frac{2 u_{v}^{A}+d_{v}^{A}}{3} & =\int_{0}^{1} d x \frac{2 u_{v}+d_{v}}{3}=1
\end{aligned}
$$

Eqs. (3.7), (3.8) are for $C^{A}=u_{v}^{A}+d_{v}^{A}=u^{A}-\bar{u}^{A}+d^{A}-\bar{d}^{A}$, respectively $C^{A}=\left(2 u_{v}^{A}-d_{v}^{A}\right) / 3$. Those express the conservation (per nucleon) of the number of valence quarks in nuclei (baryon number) and of charge.

b) For any linear combination $C=\alpha_{u} u_{v}+\alpha_{d} d_{v}$ of nuclear pdf

$$
\begin{aligned}
C^{A}\left(0, Q^{2}\right) & =C^{N}\left(0, Q^{2}\right) \\
C^{A}\left(x_{0}, Q^{2}\right) & \approx C^{N}\left(x_{0}, Q^{2}\right)
\end{aligned}
$$

By construction, Eq. (2.2) holds only for the contribution of partons from nucleons, and somehow the same is the case for (3.5). For those parts one proves the above equality for $x=0$, whereas for $0.18 \gtrsim x_{0} \gtrsim 0$, Eq. (3.10) is an approximation.

c) For any combination $x C(x)=x \sum_{i} \alpha_{i} q_{i}(x$,$) or x \sum_{i} \alpha_{i} \bar{q}_{i}(x)$

$$
\begin{aligned}
\int_{0}^{A} d x x C^{A}(x) & =\int_{0}^{A} d z z f^{P N, A}(z) \int_{0}^{1} d t t C^{N}(t) \\
\int_{0}^{A} d x x\left[u_{v}^{A}+d_{v}^{A}+2 \bar{u}^{A}+2 \bar{d}^{A}+2 s^{A}+g^{A}\right]_{x} & =\int_{0}^{A} d z z f^{P N, A}(z) * \\
\int_{0}^{1} d t t & {\left[u_{v}+d_{v}+2 \bar{u}+2 \bar{d}+2 s+g\right]_{t} } \\
\approx \int_{0}^{1} d t t & {\left[u_{v}+d_{v}+2 \bar{u}+2 \bar{d}+2 s+g\right]_{t} }
\end{aligned}
$$

Again, Eq. (3.12) is a special case, related to the momentum sumrule, which does not exactly carry over to the nuclear case. However, the peaking of the normalized $f^{P N, A}$ causes the $z$-integral in Eq. (3.11) to be very close to 1. In the case of the nuclear momentum sumrule Eq. (3.12), the deviations are really minute (see Ref. 28 for an entirely different way to mend the momentum sumrule violation in the PWIA). 
The incompleteness of the above-mentioned Eq. (2.2) for $x \lesssim 0.2$ does not in practice constitute a problem. For one, the conservation of the number of valence quarks per nucleon is guaranteed by unitarity, i.e. the normalization of $f^{P N, A}$. As the momentum sumrule (3.12) illustrates, more is requires than unitarity, in case the fact that the widths $\Delta x\left(Q^{2}\right)$ of the peaked $f$ are appreciably less than the support of $x$. Comparing the two sides of the expressions in Eq. (3.12) for 4 targets and $Q^{2}=3.5,5.0 \mathrm{GeV}^{2}$, we find differences of no more than $\approx 1 \%$.

The above sum rules involve pdf in a nucleus and in a nucleon. Various sumrules involve moments of nuclear SF and one may compare numerical results in the hadronic representation (see for instance Ref. [15, 29, 30]) and in the partonic one. For instance, Eq. (33.3) and (3.12) hint, that there exists a sumrule for $M_{-1}^{A} \equiv F_{2}^{A} / x=\sum a_{i} q_{i}^{A}$. The contributions of valence quarks is $5 / 6$, but all other parts diverge (see for instance 31 , 32). Those divergences cancel in differences of any pair of those ratios. Thus from Eqs. (3.3), (3.6) (for simplicity we disregard $\delta N / A$ corrections)

$$
\begin{aligned}
M_{-1}^{A}-M_{-1}^{A^{\prime}} & =\int_{0}^{A} \frac{d x}{x} F_{2}^{A ; N I}-\int_{0}^{A^{\prime}} \frac{d x}{x} F_{2}^{A^{\prime} ; N I} \\
& =\frac{5}{18}\left[\int_{0}^{A} d x\left(u_{v}^{A}+d_{v}^{A}\right)-\int_{0}^{A^{\prime}} d x\left(u_{v}^{A^{\prime}}+d_{v}^{A^{\prime}}\right)\right] \approx 0
\end{aligned}
$$

For all $A^{\prime} \neq A$ (including $A^{\prime}=1$ with a $x$-support, maximally different from $(0, A)$ for a target $A$ ), the upper integration limits in Eq. (3.13) are unequal. However, for the above-mentioned reason, one may neglect the contributions to the integrals in (3.13) for $x \lesssim 0.20$ and $x \gtrsim 0.95$. Hence there effectively is an approximate common upper limit $x_{U} \approx 1 \ll A, A^{\prime}$, beyond which the difference $\left(F_{2}^{A}-F_{2}^{A^{\prime}}\right)$ is negligible and the same holds for $x_{L} \lesssim 0.18$. For the pair $\mathrm{D}$, Fe and the chosen three $Q^{2}$ values, the above difference of the integrals in the hadron and pdf representations is $\approx-0.03$ [46].

Of special interest is the zeroth moment of nuclear SF, which is related to the momentum sumrule. Using Eq. (2.2)

$$
\begin{aligned}
M_{0}^{A}=\int_{0}^{A} d x F_{2}^{A ; N I}(x) & =\int_{0}^{A} d x \int_{x}^{A} d z f^{P N, A}(z) F_{2}^{\langle N\rangle}(x / z) \\
& =M_{0}^{\langle N\rangle} \int_{0}^{A} d z z f^{P N, A}(z) \approx M_{0}^{\langle N\rangle}
\end{aligned}
$$

The same moment of iso-singlet NI parts in the pdf representation is from Eqs. (3.3), (3.6) 
and (3.8) seen to be

$$
\begin{aligned}
M_{0}^{A}=\int_{0}^{A} d x F_{2}^{A ; N I}(x)= & \frac{5}{18} \int_{0}^{A} d x x\left[u_{v}^{A}+d_{v}^{A}+2\left(\bar{u}^{A}+\bar{d}^{A}\right)+\frac{4}{5} s^{A}\right]_{x} \\
& \approx \frac{5}{18}\left[1-\int_{0}^{A} d x x\left(\frac{6}{5} s^{A}+g^{A}\right)_{x}\right] \\
& \approx \frac{5}{18}\left[1-\int_{0}^{1} d x x\left(\frac{6}{5} s(x)+g(x)\right)\right]=\int_{0}^{1} d x F_{2}^{\langle N\rangle}(x)
\end{aligned}
$$

NE parts are small for the considered $Q^{2}$. When included, the normalization of $f^{P N, A}$ guarantees $M_{0}^{A, N E}=M_{0}^{N, N E}$, i.e. NE parts of $M_{0}^{A}$ are also $A$-independent.

In Figs. 2a-2e we show differences of valence, sea quark and gluon distributions functions in a nucleus and for the $p$. We choose 5 targets and display results only for $Q^{2}=5 \mathrm{GeV}^{2}$, because for the $Q^{2}$ range considered there is hardly any $Q^{2}$-dependence. Differences increase with increasing mass number, and change sign at roughly $x=0.2$ and 0.8 .

To the extent that nuclear sea and gluon distributions are close to the nucleonic ones, Eq. (3.16) shows that in the pdf representation, $M_{0}^{A}$ is practically $A$-independent. Emphasis is on the standard $\approx 50 \%$ reduction of the nucleon valence contributions $5 / 18$ due to gluons (see for instance 31), which carries over to nuclear df.

It is not feasible to directly verify the hadronic result (3.14), which requires $F_{2}^{A}$ to be known over the entire relevant $x$-range. Present data are for $x \gtrsim 0.3$ for the lowest $Q^{2}$ (for which Eq. (3.14) is not accurate) and $x \gtrsim(0.5-0.6)$ for medium $Q^{2}$. For both the missing information for $x \lesssim 0.5$ contains the major contribution to $M_{0}^{A}$. We thus take recourse to nuclear SF, computed from Eq. (2.2). Wherever data are available for the DIS $x$-range, those appear to agree very well with computed ones.

Table I displays the zeroth moments $M_{0}^{A}, A=\{\mathrm{D}, \mathrm{He}, \mathrm{C}, \mathrm{Fe}\}$, computed in the hadronic and the partonic representations (Eq. (3.14), respectively (3.15), (3.16)). Comparison of columns 2 and 3 clearly shows that the shape $f^{P N, A}$ effectively cuts the upper limit of long-range integrals at $x_{U} \approx 1.0$.

The lowest moments $M_{0}^{A}\left(Q^{2}\right)$ are seen to be dependent on $A$ in a weak and not smooth fashion. Going from D to Fe, those moments are 3-4, respectively $8 \%$ smaller than the same for the averaged nucleon $M_{0}^{\langle N\rangle}\left(Q^{2}\right)$ : D and He clearly do not follow the smooth behavior of all other nuclei. One also notices that $M_{0}^{A}$ are weakly descending functions of $Q^{2}$.

At this point we return to a verification of the result (3.14). In spite of the lacking data on $F_{2}^{A}$ for small- $x$, it is possible to reach an indirect, approximate experimental verification 
(3.14) in the hadronic representation. The method exploits knowledge on $F_{k}^{A}$ for small $x$ and the smoothness of the same in the region $x \gtrsim x_{m}, x_{m}$ being the above-mentioned, smallest $x$ measured [34, 35]. One then interpolates nuclear SFs in the intermediate region, where data are missing [25] and subsequently approximate calculates the lowest moments $M_{0}^{A}$. In spite of the fact, that the 'missing' region contributes the major part of the integrals (3.14), the moments turn out to be surprisingly close to the ones computed above. Forthcoming data from JLab experiment E03-103 [36] will enable a sharpening of the above method.

The approximate $A$-independence of the above zeroth moments of any pair of SF entails the same for the differences

$$
M_{0}^{A}-M_{0}^{A^{\prime}}=\int_{0}^{A} d x F_{2}^{A ; N I}-\int_{0}^{A^{\prime}} d x F_{2}^{A^{\prime} ; N I} \approx \int_{x_{0}}^{x_{U}} d x\left[F_{2}^{A ; N I}-F_{2}^{A^{\prime} ; N I}\right] \approx 0
$$

The vanishing of the difference is attributed to about equal effective $x$-ranges $\left(x_{0}, x_{U}\right) \approx$ (0.15,0.85), which replace actual unequal supports [37]. Eq. (3.17) implies that in the above common interval, the difference of two SF has to change sign at least once, or in different terms: the generalized EMC ratios $\mu^{A, A^{\prime}}\left(x, Q^{2}\right)=F_{2}^{A}\left(x, Q^{2}\right) / F_{2}^{A^{\prime}}\left(x, Q^{2}\right)$ passes the value 1 in the above $x$-interval, as is indeed observed for all $A, Q^{2}$ (see Ref.[38] for a discussion in an entirely different context).

The simplest cause for approximate $A$-independence of $M_{0}^{A}$ would be the same for $F_{2}^{A}$, but that appears not to be the case. In the dominant classical region $x \lesssim 0.90$, differences $F_{2}^{A}-F_{2}^{A^{\prime}}$ in both representations grow with $x$ beyond $\approx 0.18$ and may become as large as $50-60 \%$, which is far larger than the spread in $M_{0}^{A}$ (cf. Table I). We shall shortly return to this point.

\section{EMC RATIOS IN THE PARTON DISTRIBUTION FUNCTIONS REPRE- SENTATION.}

We have computed $F_{2}^{A, N I}$ in the pdf representations, using Eq. (3.3), (3.5) and (3.6). To those we added the NE components (2.7), which are only relevant for $|1-x| \lesssim 0.05$. The total EMC ratios $F_{2}^{A} / F_{2}^{D}$ are then compared with recently determined counterparts in the hadron representation [38]. For the range $0.2 \lesssim x \lesssim 1.2$ those, and avaibale data for He, Fe and $\mathrm{Au}$ [39, 40, 41], are shown in Figs. 4a,b for $Q^{2}=3.5,5.0 \mathrm{GeV}^{2}$.

Up to $x \lesssim 0.65$ there is close agreement between the computed ratios. Beyond that 
point deviations set in, which grow while pdf $\gg$ hadr. The latter situation is reversed for $x \gtrsim 0.90$. Both representations overestimate the relative maxima in $\mu^{A}$ around $x=0.9$, but over the entire $x$-range, the hadronic results are closer to the data than those for the pdf. We attribute this to inferior $F_{2}^{n}$ input for larger $x$, which propagates into $F_{2}^{A}$ through the use of Eq. (2.2). Also of interest are the slightly lower pdf results for $x \lesssim 0.65$ and the much higher ones beyond up to $x \approx 0.9$.

The apparent insensitivity of EMC ratios $\mu^{A}$ to the representation, in spite of the large differences in the participating $F_{2}^{A}$, combined with points a), b) in Section III suggest the following: Irrespective of the cause of the dependence of nuclear SF on $A$ and/or representation, the approximate independence of the zeroth moments (3.14) on both, forces the differences in $F_{2}^{A}$ in the regions $x \lesssim 0.18$ and $0.18 \lesssim x \lesssim 0.90$ to be nearly balanced. Consequently, if EMC ratios in one area are in some way ordered in $A$, that ordering is inverted in the second one. It almost seems that deviations of EMC ratios from 1 can be generated by an integral-preserving, affine transformation with $x \approx 0.18,0,90$ as fixed-points, and having in particular for $A \lesssim 12$, a characteristic $A$-dependence, and having $x \approx 0.18,0,90$ as fixed-points.

Finally, out of sheer curiosity we followed the pdf results down to $x=10^{-5}$. Results for $F_{2}^{A}$ hardly change from their $A$-independent values around $x=0.15$, causing EMC ratios to stay close to 1 for decreasing $x$. The above is actually observed down to $x \approx 10^{-3}$. Only for the smallest $x$ do screening effects deplete $\mathrm{df}$ and cause $\mu^{A}$ to slowly reach values $\approx 0.6-0.7$.

\section{COMPARISON, CONCLUSIONS.}

In this note we defined $\mathrm{df}$ for partons in a nucleus, using for the latter a relation between nucleonic and nuclear hadron SF without any adjustable parameter. We showed that either

exactly or very closely, those respect basic sumrules. Observables such as $F_{2}^{A}$ may be expressed in either representation, and by construction produce in principle the same $F_{2}^{A}$ for identical input $F_{2}^{p, n}$.

We have shown that this is not the case in practice, specifically not for $F_{2}^{n}$. The pdf choice rests on the 'primitive' approximation $F_{2}^{n}=2 F_{2}^{D}-F_{2}^{p}$, which is satisfactory for $x \lesssim 0.3$, but deteriorates with increasing $x$, while a well-founded extraction method has been used in the hadronic representation. Consequently EMC ratios, computed in the two versions, 
practically coincide for $x \lesssim 0.65$. For large $x$ deviations appear, which reflect the similarly deviating $F_{2}^{n}$ from a better founded extracted function: the hadronic representation of EMC ratios produces the better fits to the data.

The above, and the fact that deviations of EMC ratios from 1 appear in distinct areas to be balanced for all $A$, seems linked to the lowest moment $M_{0}^{A}$ of $F_{2}^{A}$ : those are for any $Q^{2}$ practically independent of $A$.

We continued pdf calculations down to the smallest $x$, which is the region were the above criticism does not hold. However, there Eq. (3.5) misses primarily (anti-)screening effects. Nevertheless, down to $x \approx 10^{-3}$ the agreement with data persists, but the pdf results cannot describe anti-screening depletion of df in $\mu^{A}$ for the smallest measured $x$.

Our almost natural choice of distribution functions of partons in a nucleus is clearly one out of many possible ones, and we shall mention a few suggested alternatives. For instance Eskola etal address participating nuclear SF in EMC ratios, which are generated from parametrized input for a reference $Q_{0}^{2}$, and which are subsequently evolved to the desired $Q^{2}$ [5]. Parameters are constrained, for instance by fixing the average position of minima of EMC ratios. Data determine those parameters.

Next we mention Kumano and co-workers who, in spite of different $x$-support for $F^{A}$ and $F^{N}$, assume a linear relation between df for partons in a nucleus and in a nucleon 6]. The species-dependent, relating weight functions $w_{i}(x, Z, A)$ contain parameters for a scale $Q_{0}^{2}$ and the resulting df of partons in a nucleus are again evolved to any desired $Q^{2}$, ultimately producing parametrized nuclear SF and EMC ratios. A large number of adjustable parameters leads to fits from the smallest $x$ up to $x \leq 1$. With the connecting weight function having no meaning beyond $x=1$, the interesting region $x \geq 1$ is out of reach in that approach. A more serious drawback of the method may be the lack of physical meaning of the weight functions $w_{i}$ and its parameters.

Finally we discuss approaches, where df of partons in a nucleus are those for a nucleon bound in scalar and vector mean fields [8, 9], which couple to quarks in a nucleon [42]. Off-hand, the above and our phenomenological approach seem to have little in common, but this is actually not the case, and it is instructive to trace the connection. We recall that the original proposal Eq. (2.2) was inspired by a model, where valence quarks in a nucleus cluster in bags [11]. Total interactions between quarks in two different bags have there been replaced by phenomenological $N N$ forces, acting on the centers of those bags, thus replacing 
quark dynamics by those for hadrons. The above treatment of a nucleus partially calls back quark degrees of freedom, and are from Eq. (3.4) seen to mix the latter with hadronic degrees of freedom through the $\mathrm{SF} f^{P N, A}$.

In contrast, the hybrid meson-quark coupling model, where the interactions of a single quark in a given nucleon with all quarks in the remaining $A-1$ bags are replaced by mean fields. As expected from its intermediate position, it is possible to sum in that model the above meson-quark interactions over the valence quarks in a bag, and to construct a $N N$ interaction with many-body components, mediated by the same mean boson fields. This has recently been shown to be possible [43].

The above has been applied to nuclear matter [8, 9] and it is clearly of interest to see applications of finite nuclei. A first example is a treatment of ${ }^{3} \mathrm{He}$ in the PWIA [10] and one should look forward to results for higher $A$.

[1] S.V. Akulinichev, S.A. Kulagin and G.M. Vagradov, Phys. Lett. B 158, 485 (1985).

[2] E.L. Berger and F. Coester, Ann. Rev. of Nuclear and Particle Science, Vol. 37, 463 (1987).

[3] R.P. Bickerstaff, M.C. Birse and G.A. Miller, Rep. 40048-N5, University of Washington, Seattle.

[4] L.L. Frankfurt and M.I. Strikman, Phys, Report 160, 235 (1988).

[5] K.J. Eskola, V.J. Kolhinen and P.V. Ruuskanen, Nucl. Phys. B 535 (1998).

[6] M. Hirai, S. Kumano and M. Miyama, Phys. Rev. D 64, 034003 (2001); M. Hirai, S. Kumano and T.-Nagai , Phys. Rev. D 70, 044905 (2004).

[7] C.J. Benesh, T. Goldman and G.J. Stephenson Jr., Phys. Rev. C 68, 045208. (2203).

[8] H. Mineo, W. Bentz, N. Ishii, A.W. Thomas and K. Yazaki, Nucl. Phys. A 735, 482 (2004).

[9] F.M. Steffens, A.W. Thomas and K. Tsushima, arXiv:hep-ph/0405096

[10] S. Scopetta, Phys. Rev. C 70, 015205 (2004).

[11] S.A. Gurvitz and A.S. Rinat, TR-PR-93-77/ WIS-93/97/Oct-PH; Progress in Nuclear and Particle Physics, Vol. 34, 245 (1995).

[12] H. Gersch, L.J. Rodriguez and Phil N. Smith, Phys. Rev. A 5, 1347 (1973).

[13] S.A. Gurvitz and A.S. Rinat, Phys. Rev. C 65, 024310 (2002).

[14] A.S. Rinat and M.F. Taragin, Phys. Rev. C 60, 044001 (1999). 
[15] A.S. Rinat and M.F. Taragin, Phys. Rev. C 62, 034602 (2000).

[16] A.S. Rinat and M.F. Taragin, Nucl. Phys. A 598,349 (1966); ibid. A 620, 412 (1997); Erratum: ibid. A 623, 773 (1997); Phys. Rev. C 0, 04461 (1999).

[17] A.S. Rinat and M.F. Taragin Phys. Rev. C 65, 044601(R) (2001).

[18] M. Viviani, A. Kievsky and A.S. Rinat, Phys. Rev. C 67, 034003 (2003).

[19] C.H. Llewelyn Smith, Phys. Lett. B 128 (1983) 107; M. Ericson and A.W. Thomas, ibid p. 112 .

[20] S.A. Kulagin, G. Piller and W. Weise, Phys. Rev. C 50, 1154 (1994); G. Piller and W. Weise, Phys. Report 330, 1 (2000).

[21] G.B. West, Ann. of Phys. (NY) 74, 646 (1972); W.B. Atwood and G.B. West, Phys. Rev. D7, $773(1973)$

[22] H.L. Lai et al, Eur. Phys. J. C 12, 375 (2000) and extension CTEQ6, [ArXiv: JHEP]

[23] M. Arneodo, Phys. Lett. B 364, 107 (1995).

[24] E. Christy etal, Phys. Rev. C 70, 015206 (2004).

[25] A.S. Rinat and M.F. Taragin, Phys. Lett. B 551, 284 (2003).

[26] H. Budd, A. Bodek and J. Arrington, ArXiv:hep-ex/0308005 ].

[27] N. Armesto et al, Eur. Phys. J. C 29, 531 (2003).

[28] J. Rozynek and G. Wilk, ArXiv:nucl-th/0406045 ].

[29] L.F. Abbott and R.M. Barnett, Ann. Phys. (N.Y.) 125, 276 (1980); M.R. Pennington and G.G. Ross, Nucl. Phys. B 179, 324 (1981).

[30] C.S. Armstrong et al., Phys. Rev. C63, 094008 (2001).

[31] R.G. Roberts, The structure of the proton, DIS, Cambridge University Press (1990).

[32] R.S. Towell et al., Phys. Rev. D 64, 052002 (2001).

[33] V. Guzey et al, Phys. Rev. D 64054503 (2001).

[34] J. Arrington etal., Phys. Rev. Lett. 82, 2056 (1999): J. Arrington, PhD Thesis Cal.Tech, 1998.

[35] I. Niculescu et al., Phys. Rev. Lett. 85, 1182 (2000).

[36] J. Arrington, D. Gaskal et al., JLab experiment E03-103.

[37] In his review article Phys. Rep. 240, 301 (1944), Arneodo refers to his PhD thesis, where a related difference $\approx \int_{0}^{2} d x\left[F_{2}^{A}-F_{2}^{D}\right] \approx 0$ is considered, i.e. in the harmless approximation $A \rightarrow x_{U} \rightarrow 2$. 
TABLE I: Zeroth moments of $F_{2}^{A}$ in the hadron (h) and a parton (p) representation, with upper limits $A$ approximated by 1 and 2 .

\begin{tabular}{|lr|l|l|}
\hline \multicolumn{2}{|c|}{ target } & \multicolumn{1}{|c|}{$\int_{0}^{1} d x F_{2}^{A}\left(x, Q^{2}\right)$} & \multicolumn{1}{c|}{$\int_{0}^{2} d x F_{2}^{A}\left(x, Q^{2}\right)$} \\
\hline $\mathrm{D}$ & $\mathrm{h}$ & $0.1492,0.1484,0.1409$ & $0.1493,0.1484,0.1409$ \\
& $\mathrm{p}$ & $0.1505,0.1470,0.1413$ & $0.1506,0.1470,0.1413$ \\
\hline${ }^{4} \mathrm{He}$ & $\mathrm{h}$ & $0.1455,0.1450,0.1378$ & $0.1459,0.1453,0.1379$ \\
& $\mathrm{p}$ & $0.1464,0.1433,0.1378$ & $0.1467,0.1435,0.1379$ \\
\hline $\mathrm{C}$ & $\mathrm{h}$ & $0.1434,0.1434,0.1370$ & $0.1440,0.1439,0.1372$ \\
& $\mathrm{p}$ & $0.1430,0.1403,0.1353$ & $0.1434,0.1408,0.1383$ \\
\hline $\mathrm{Fe}$ & $\mathrm{h}$ & $0.1402,0.1403,0.1338$ & $0.1406,0.1406,0.1339$ \\
& $\mathrm{p}$ & $0.1447,0.1403,0.1353$ & $0.1433,0.1405,0.1351$ \\
\hline$\langle N\rangle$ & $\mathrm{h}$ & $0.1554,0.1510,0.1438$ & \\
& $\mathrm{p}$ & $0.1510,0.1475,0.1420$ & \\
\hline
\end{tabular}

[38] A.S. Rinat, M.F. Taragin and M. Viviani, ArXiv:nucl-th/0412053.

[39] J. Gomez etal., Phys. Rev. D 49, 4348 (1994).

[40] A. Bodek et al., Phys. Rev. Lett. 50, 1431 (1983); A.C. Benvenuti et al Phys. Lett. B 189, 483 (1987); S. Dasu et al., ibid. 60, 2591 (1988).

[41] P. Amadrauz et al., Nucl. Phys. B 441, 3,12 (1995).

[42] P.A.M. Guichon, Phys. Lett. B 200, 235 (1988); P.A.M. Guichon et al, Nucl. Phys. A 601, 349 (1996).

[43] P.A.M. Guichon and A.W. Thomas, [arXiv: nucl-th/0402064.

[44] We also disregard mixing of nucleonic SF, the relative importance of which diminishes with increasing $Q^{2}[21]$.

[45] Lacking experimental information on input elements (e.g. $G_{E}^{n}$ ) for higher $Q^{2}$, we occasionally use extrapolations from lower $Q^{2}[26]$.

[46] A special case occurs for members of an iso-doublet $A=A^{\prime}$, for which Eq. (3.13) is a generalized Gottfried sum. For a recent discussion of its apparent shortcomings, we refer to Ref. 33]. 


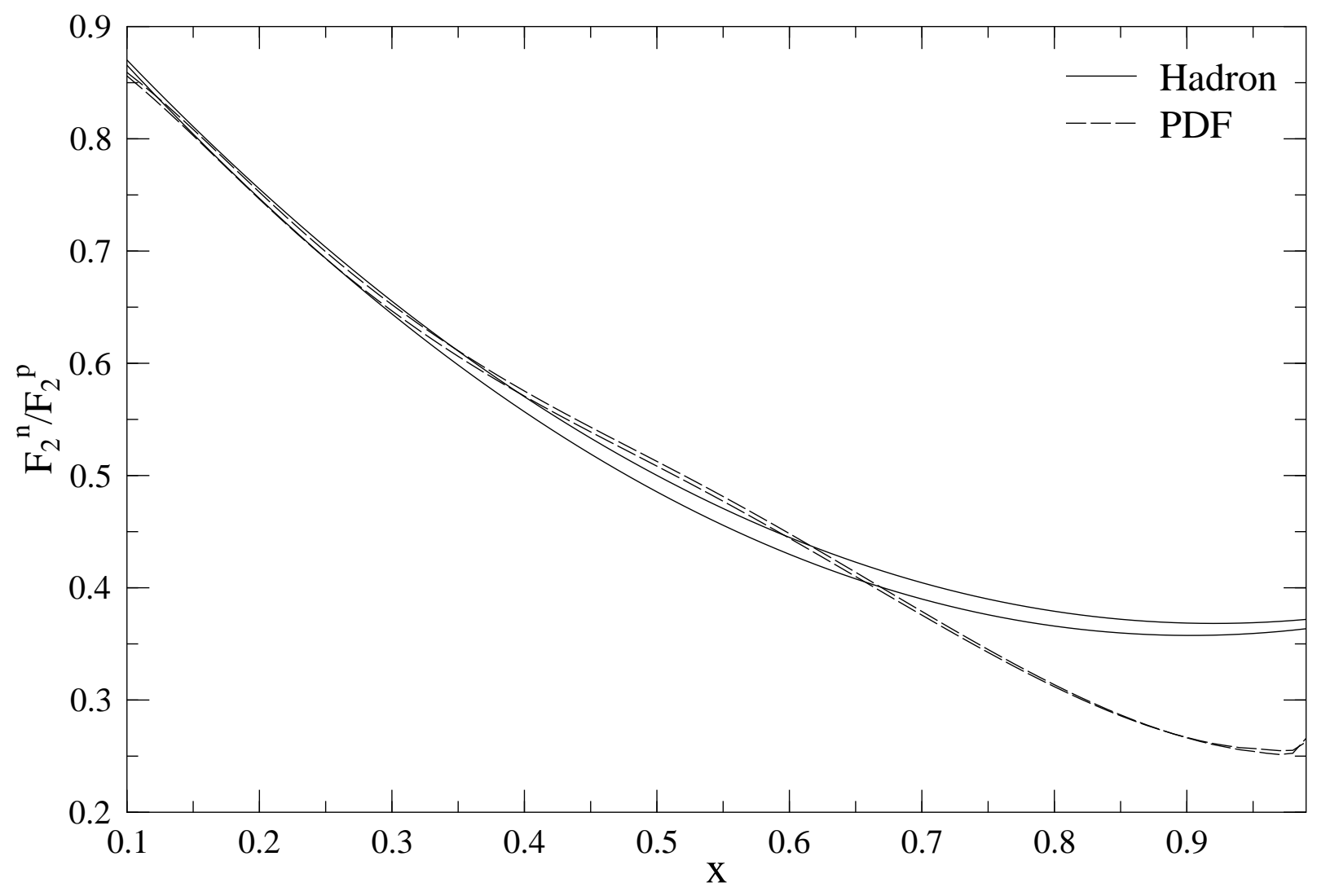

FIG. 1: The ratio $F_{2}^{n}\left(x, Q^{2}\right) / F_{2}^{p}\left(x, Q^{2}\right)$ in the hadronic (drawn lines) and the pdf representation (dashes); Upper and lower curves correspond to $Q^{2}=3.5,5.0 \mathrm{GeV}^{2}$. 


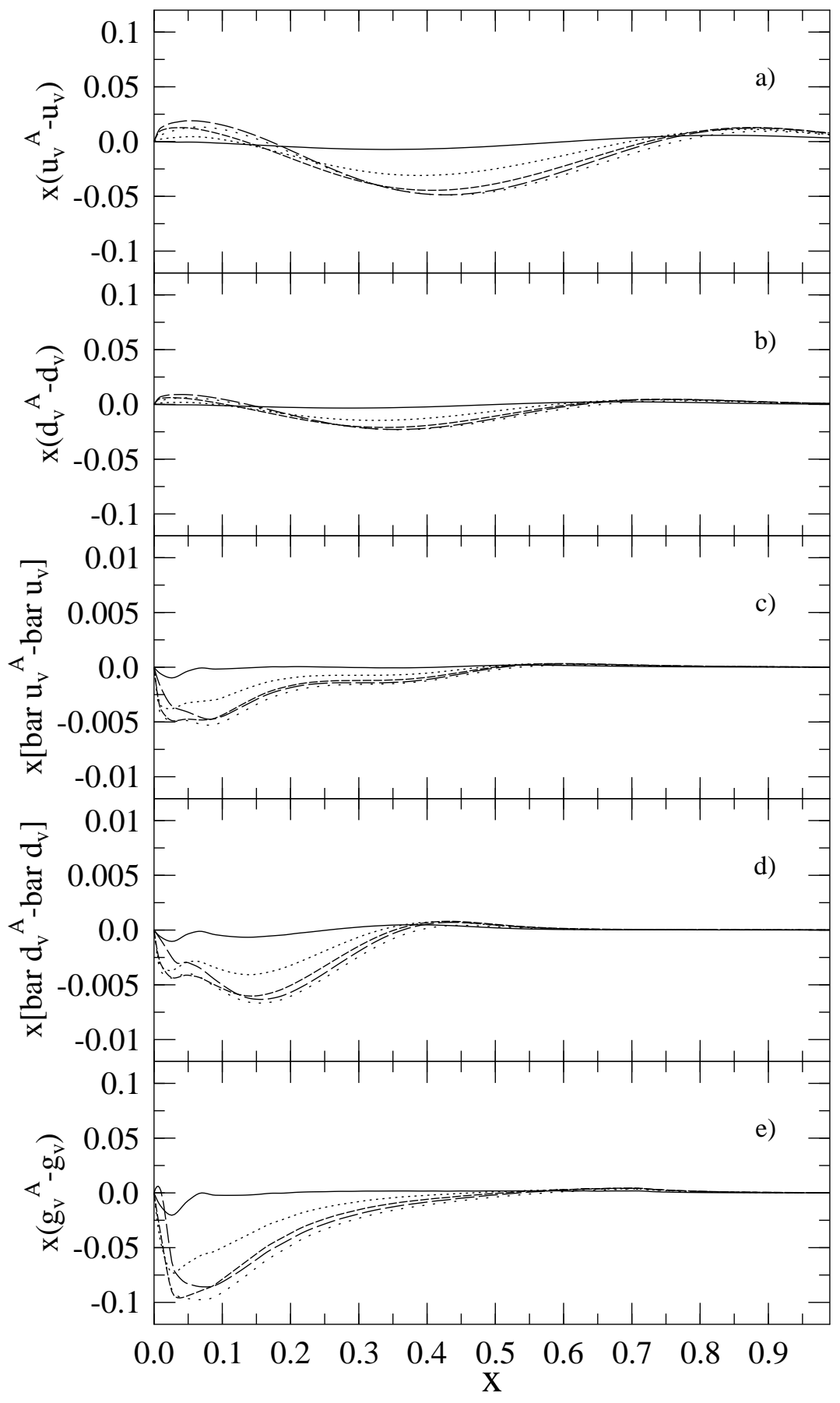

FIG. 2: Differences $x\left(q^{A}-q\right)$ for D (drawn line), ${ }^{4} \mathrm{He}$ (dots), C (spaced dots), Fe (short dashes) and $\mathrm{Au}$ (long dashes) and a $p ; Q^{2}=5 \mathrm{GeV}^{2}$. a): $q=u_{v}$; b) $q=d_{v}$; c) $q=\bar{u}$; d) $q=\bar{d}$; e) $q=g$. 


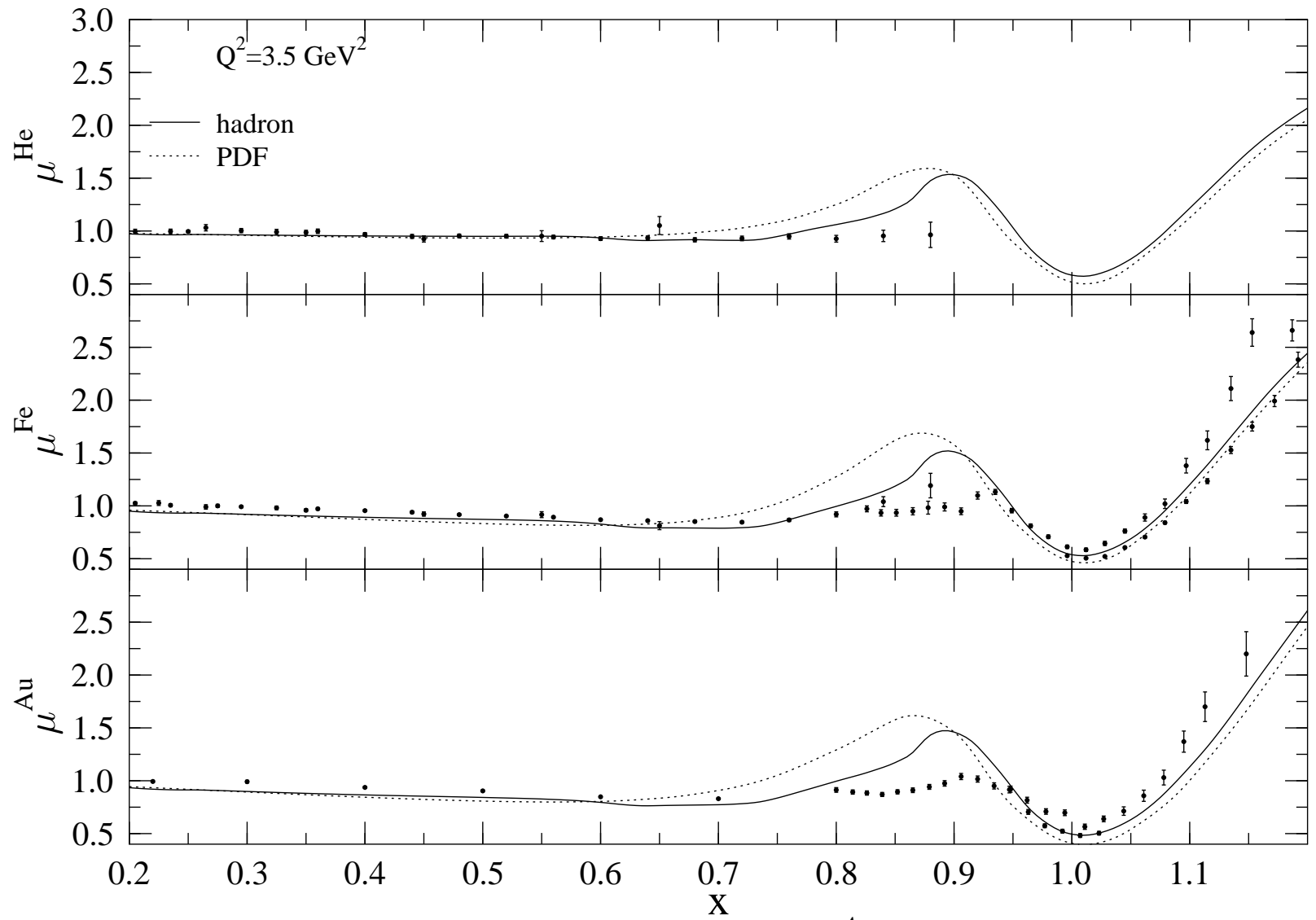

FIG. 3: Hadronic and pdf representations of EMC ratios $\mu^{A}$ (drawn and dotted lines), $\mathrm{A}=\mathrm{He}, \mathrm{C}$ and Fe for $0.2 \lesssim x \lesssim 1,2, Q^{2}=3.5 \mathrm{GeV}^{2}$. Data are from [39, 40, 41]. 


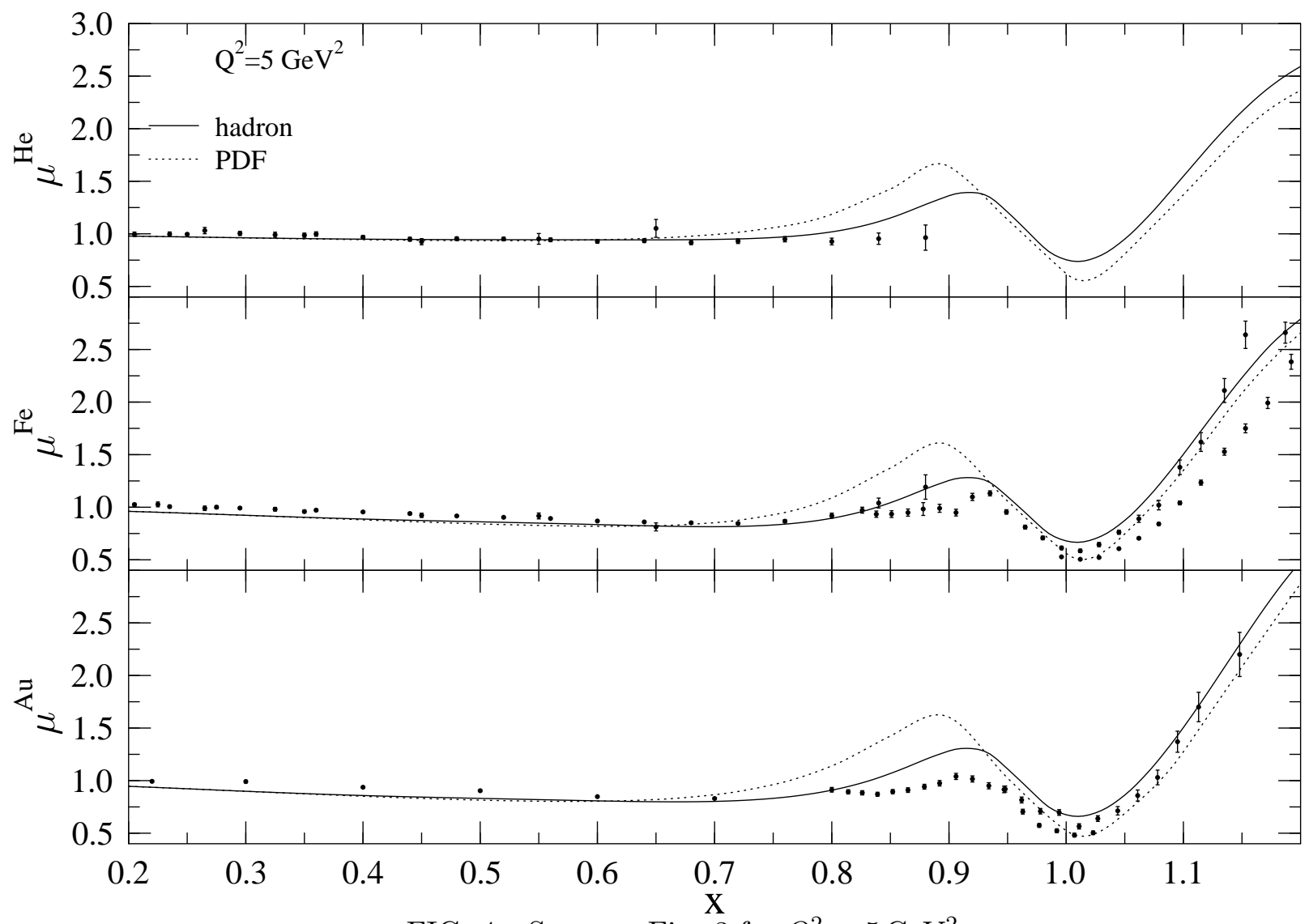

FIG. 4: Same as Fig. 3 for $Q^{2}=5 \mathrm{GeV}^{2}$. 